\title{
Pathologic N1 non-small cell lung cancer: Correlation between pattern of lymphatic spread and prognosis
}

\author{
Alessandro Marra, MD, PhD \\ Ludger Hillejan, MD \\ George Zaboura, MD \\ Toshio Fujimoto, MD \\ Dieter Greschuchna, MD \\ Georgios Stamatis, MD
}

From the Department of Thoracic Surgery and Endoscopy, Ruhrlandklinik, Essen, Germany.

Presented at the Tenth Annual Meeting of the German Society of Thoracic Surgery (DGT, Deutsche Gesellschaft für Thoraxchirurgie), Berlin, June 7-9, 2001.

Received for publication March 4, 2002; revisions requested April 23, 2002; revisions received May 30, 2002; accepted for publication June 5, 2002.

Address for reprints: Alessandro Marra, MD, Department of Thoracic Surgery and Endoscopy, Ruhrlandklinik, Tüschener Weg 40-45239 Essen, Germany (E-mail: alexmarra@yahoo.it).

J Thorac Cardiovasc Surg 2003;125:543-53

Copyright $(9) 2003$ by The American Association for Thoracic Surgery

$0022-5223 / 2003 \$ 30.00+0$

doi: $10.1067 / \mathrm{mtc} .2003 .322$
Objectives: Patients with N1 non-small cell lung cancer represent a heterogeneous population with varying long-term survivals. Prognosis and pattern of recurrence seem to be particularly affected by the level of lymph node involvement.

Methods: From 1990 to 1995, a total of 1954 consecutive patients underwent surgical resection for non-small cell lung cancer: 549 (28\%) had ipsilateral pulmonary lymph node metastases $(\mathrm{N} 1)$. The hospital survivors $(\mathrm{n}=535)$ were reviewed. Three levels of lymph node metastases (hilar, interlobar, and lobar) were identified according to the new Regional Lymph Node Classification for Lung Cancer Staging and differentiated from lymph node involvement on the basis of direct invasion.

Results: The overall 5-year survival of patients with N1 disease was $40 \%$. Survival was related in the univariate analysis to T classification, level-type of N1 involvement, number of involved nodes, multilevel involvement, Karnofsky Index, R status, and adjuvant therapy. In the multivariate analysis, only $\mathrm{T}$ classification and level-type of N1 involvement clearly showed statistical power $(P=.000$ and $P=$ .001 , respectively). The pattern of cancer relapse according to level-type of N1 involvement differed significantly: hilar N1 disease recurred at distant sites in $41 \%$ of patients and locoregionally in $12 \%$ of patients, whereas N1 disease by direct invasion occurred in $24 \%$ and $17 \%$ of patients, respectively $(P=.030)$.

Conclusions: Metastases to ipsilateral hilar, interlobar, or both, lymph nodes are associated with a poorer prognosis compared with metastases in intralobar lymph nodes or with lymph node involvement by means of direct invasion. Although surgical resection remains the mainstay of treatment, the high rate of tumor recurrence in both groups mandates further randomized studies with multimodality therapy approaches.

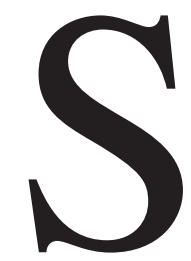

ince the publication of the PORT meta-analysis in $1998,{ }^{1}$ which showed a detrimental effect or no effect of adjuvant radiotherapy on survival of patients with completely resected early-stage non-small cell lung cancer (NSCLC), it was our concern to better understand the biologic behavior of early-stage NSCLC and to develop new therapeutic strategies able to improve the prognosis of patients with this disease.

Particularly, NSCLC with metastases to the pulmonary (N1) lymph nodes shows a disappointing prognosis for an early-stage tumor: approximately $40 \%$ overall survival at 5 years compared with approximately $60 \%$ for patients with N0 disease. According to the pathologic staging, 5-year survival was 55\% for patients with stage IIA pT1 N1 disease, 39\% for patients with stage IIB pT2 N1 disease, 25\% for 
TABLE 1. Clinical features of 535 patients with pN1 NSCLC (1990-1995)

\begin{tabular}{|c|c|c|}
\hline & No. & $\%$ \\
\hline \multicolumn{3}{|l|}{ Age, y } \\
\hline Range & $34-84$ & \\
\hline Median & 60.9 & \\
\hline \multicolumn{3}{|l|}{ Sex } \\
\hline Male/female & $454: 81$ & $85: 15$ \\
\hline \multicolumn{3}{|l|}{ Symptoms } \\
\hline Asymptomatic & 149 & 33 \\
\hline Cough & 147 & 32 \\
\hline Hemoptysis & 68 & 15 \\
\hline Chest pain & 56 & 12 \\
\hline Other & 39 & 8 \\
\hline \multicolumn{3}{|l|}{ Lobe } \\
\hline RUL & 155 & 29 \\
\hline $\mathrm{ML}$ & 23 & 4 \\
\hline RLL & 94 & 18 \\
\hline LUL & 162 & 30 \\
\hline LLL & 101 & 19 \\
\hline \multicolumn{3}{|l|}{ Location } \\
\hline Central & 377 & 70 \\
\hline Peripheral & 158 & 30 \\
\hline \multicolumn{3}{|l|}{ Clinical stage } \\
\hline 1 & 297 & 55.5 \\
\hline II & 131 & 24.5 \\
\hline III & 72 & 13.5 \\
\hline IV & 2 & 0.5 \\
\hline Unknown & 33 & 6.0 \\
\hline \multicolumn{3}{|l|}{ Karnofsky Index } \\
\hline $100 \%$ & 76 & 14 \\
\hline $90-80 \%$ & 339 & 63 \\
\hline$\leq 70 \%$ & 79 & 15 \\
\hline \multirow{2}{*}{\multicolumn{3}{|c|}{$\mathrm{FEV}_{1}$}} \\
\hline & & \\
\hline$>2.0 \mathrm{~L}$ & 319 & 59 \\
\hline $1.5-2.0 \mathrm{~L}$ & 155 & 29 \\
\hline$<1.5 \mathrm{~L}$ & 31 & 6 \\
\hline Lost data & 30 & 6 \\
\hline \multirow{2}{*}{\multicolumn{3}{|c|}{$\begin{array}{l}\mathrm{FEV}_{1} \% \\
\text { predicted }\end{array}$}} \\
\hline & & \\
\hline$>70 \%$ & 248 & 46 \\
\hline $50-70 \%$ & 205 & 38 \\
\hline$<50 \%$ & 52 & 10 \\
\hline Lost data & 30 & 6 \\
\hline
\end{tabular}

patients with stage IIIA pT3 N1 disease, and approximately $8 \%$ for patients with stage IIIB pT4 N1 disease. ${ }^{2}$

Data from the literature suggest a difference in the longterm outcome between cancers metastasized to the hilar and lobar lymph nodes, with the former group being considered as representing an early N2 disease and the latter as representing disease that is not yet spreading. ${ }^{3-7}$

For this purpose, we retrospectively reviewed our population of operated patients with pathologic N1 NSCLC disease to assess whether survival is influenced by level and type of lymph node involvement and to investigate the effectiveness of our previous therapeutic concepts for this group of patients.

\section{Patients and Methods}

Between 1990 and 1995, 1954 consecutive patients underwent surgical resection for NSCLC at the Department of Thoracic Surgery and Endoscopy of the Ruhrlanklinik, Essen, Germany. Five hundred forty-nine (28\%) patients had involvement of the ipsilateral pulmonary lymph nodes and were classified as having pathologic N1 disease. Among them, we observed a 30-day mortality of $2.5 \%$ (14 patients), whereas there were 535 hospital survivors suitable to our prognostic analysis.

In this group the median age was 60.9 years (range, 34-84 years). The male/female distribution was 5.7:1 (454/81) and reflects the relative incidence of risk factors (eg, cigarette smoking and exposure to industrial chemicals) in the general population of Germany until the early 1990s.

One third of these cancers was diagnosed on routine chest radiography, and cough, hemoptysis, and pain were leading revealing symptoms in $32 \%, 15 \%$, and $12 \%$ of patients, respectively. The median duration time of symptoms was 12 weeks (range, 1-100 weeks).

Before the operation, all patients underwent a thorough staging with chest radiography, chest and upper abdomen (and/or abdomen sonography) computed tomography (CT), bone scanning, bronchoscopy, and cervical mediastinoscopy, as well as routine cardiopulmonary function testing.

According to the revised International System for Staging Lung Cancer of the International Union Against Cancer (UICC) and the Regional Lymph Node Classification for Lung Cancer Staging, ${ }^{2,8}$ clinical stage I disease was found in 297 (55.5\%) patients, stage II disease in $131(24.5 \%)$ patients, stage III disease in $72(13.5 \%)$ patients, and stage IV disease in $2(0.5 \%)$ patients; the clinical stage was not reported in 33 records.

Patient characteristics and clinical presentation of the primary tumor are listed in Table 1.

The operation included, in all but 2 patients, the anatomic resection of the involved lobe or lung and a systematic lymph node dissection (upper and lower mediastinal compartments, as well as subcarinal lymph nodes). The indication to pneumonectomy was a centrally located tumor or interlobar-hilar lymph node metastasis with proved extranodal tumor growth, lymphangiosis, or both on frozen section.

A pneumonectomy was performed in 243 (45.5\%) patients, a sleeve pneumonectomy in $8(1.5 \%)$ patients, a bilobectomy in 37 (6.6\%) patients, a lobectomy in $196(36.7 \%)$ patients, a sleeve lobectomy in $49(9.3 \%)$ patients, and a segmentectomy in $2(0.4 \%)$ elderly patients with severe comorbidity.

A postoperative pathologic finding of hilar-interlobar extranodal metastases or bronchial R1 resection lead, if functionally suitable, to immediate completion pneumonectomy in $9(1.7 \%)$ patients and $1(0.2 \%)$ patient, respectively. Our criteria to perform a completion pneumonectomy are described elsewhere. ${ }^{9}$

After definitive surgical treatment, complete resection (R0) was achieved in $436(82 \%)$ patients; a microscopic involvement of resection margins (R1) was found in $75(14 \%)$ patients, whereas gross tumor residual masses (R2) could not be resected in $22(4 \%)$ patients. Anatomic R1 distribution was as follows: hilar fat tissue in 46 patients, bronchial stump or peribronchial tissue in 15 patients, mediastinal fat in 4 patients, interlobar surface of the resected lobe in 1 patient, and chest wall in 6 patients. As concerns 
the $\mathrm{R} 2$ resections, macroscopic residual tumors were located in the mediastinal fat in 11 patients, in the wall of the great vessels in 5 patients, in a vertebral body in 1 patient, in the bronchial stump in 2 patients, and in the chest wall in 3 patients.

Surgical complications are listed in Table 2.

\section{Primary Tumor}

The tumor was classified according to the World Health Organization International Histological Classification of Tumours ${ }^{10}$ as squamous cell carcinoma (SCC) in $348(65 \%)$ patients, as adenocarcinoma in $139(26 \%)$ patients (including 8 bronchioloalveolar carcinomas), as anaplastic large cell carcinoma in $26(5 \%)$ patients, as adenosquamous carcinoma in $18(3 \%)$ patients, and as other types in $4(1 \%)$ patients. This distribution differs from the entire cohort of 1954 patients with NSCLC because SSC was observed in $55 \%$ of patients and adenocarcinoma in more than one third $(37 \%)$ of patients. As concerns the grading, the tumor was well differentiated (G1) in $29(5 \%)$ patients, moderately differentiated (G2) in $283(53 \%)$ patients, poorly differentiated (G3) in 193 $(36 \%)$ patients, and undifferentiated (G4) in $26(5 \%)$ patients; the grading was unknown in $4(1 \%)$ patients. A lymphatic or blood vessel invasion was observed in 138 (26\%) patients. For the pathologic $\mathrm{T}$ classification of the primary tumor, we retrospectively followed the revised International System for Staging Lung Cancer of the UICC2: 67 (12\%) tumors satisfied the criteria for T1, 287 (54\%) for T2, 128 (24\%) for T3, and 53 (10\%) for T4 disease.

\section{Lymph Node Metastases}

We revised the pathologic description of the anatomic location of lymph node metastases according to the recently published Regional Lymph Node Classification for Lung Cancer Staging. ${ }^{8}$ Our pathologists described as "direct invasion of lymph nodes" an infiltrative extension of viable tumor cells without loss of continuity from the primary tumor mass to the lymphatic tissue of an adjacent node. A connection by lymphangiosis carcinomatosa between the involved lymph node and the primary tumor has not been considered direct invasion.

One hundred twenty-one (23\%) patients had metastases in the hilar lymph nodes (N1h, station 10) as a more advanced level, 76 $(14 \%)$ patients in the interlobar nodes (N1i, station 11), and 87 $(16 \%)$ patients in the peripheral intralobar nodes (N1p, stations 12 , 13, and 14), whereas in 251 (47\%) patients the lymph node involvement was through direct extension of the primary tumor on either hilar, interlobar, or lobar nodes. A multilevel involvement was observed in $84(69 \%)$ patients of the N1h group and in 45 (59\%) patients of the N1i group. Metastasized tumor cells skipped intralobar, interlobar, or both lymph node stations in $70(13 \%)$ patients.

Adjuvant radiotherapy was administered to 191 (36\%) patients with either $\mathrm{pN} 1 \mathrm{~h}$ disease after lobectomy or R1-2 resection margins; the median radiation dose was 55 Gy. Two $(0.5 \%)$ patients underwent adjuvant chemoradiotherapy. Metastases to the interlobar and intralobar nodes and direct lymph node infiltration at any level were not considered indications for any adjuvant treatment. Patients who underwent an R0 pneumonectomy for tumor with hilar node metastasis also did not receive radiotherapy.

The median follow-up time was 36.5 months (range, 1-133 months). One hundred (18.7\%) patients had a follow-up of 1 to 12
TABLE 2. Surgical complications after 535 thoracotomies for N1 NSCLC

\begin{tabular}{lrr}
\hline & No. & $\%$ \\
\hline Major complications & & \\
Bronchopleural fistula-empyema & 15 & 3 \\
Pneumonia & 14 & 3 \\
Heart failure-infarction & 13 & 2 \\
Rethoracotomy for bleeding & 11 & 2 \\
Respiratory failure-ARDS & 5 & 1 \\
Other & 5 & 1 \\
Minor complications & & \\
Cardiac arrhythmia & 135 & 25 \\
Prolonged air leak & 55 & 10 \\
Pleural effusion & 8 & 1 \\
Wound infection & 6 & 1 \\
Other & 46 & 9
\end{tabular}

ARDS, Adult respiratory distress syndrome.

months, $220(41.1 \%)$ had a follow-up of 13 to 60 months, 173 (32.3\%) had a follow-up of 61 to 120 months, and $33(6.2 \%)$ had a follow-up of more than 120 months. Nine (1.7\%) patients were lost to follow-up.

\section{Statistical Analysis}

Cancer recurrence was carefully divided into 2 categories according to the site of initial relapse: locoregional or distant. Locoregional recurrence was defined as any recurrent disease within the ipsilateral hemithorax, mediastinum, or supraclavicular lymph nodes. All other sites of recurrence were considered distant metastases (M). The cause of death was recorded as either cancer related, other disease, or unknown. Cross-tabulations with the Pearson $\chi^{2}$ test were used for group comparison when appropriate. Survivals were calculated by using the Kaplan-Meier life-table method, ${ }^{11}$ and survival curve comparisons were made by using a log-rank test, ${ }^{12}$ in which the initial day of treatment was the day of the operation. Multivariate analysis was based on the Cox proportional hazards model. ${ }^{13}$ Analysis was carried out by using a software package (Statistical Program for the Social Sciences, release 10.0.7, 2000; SPSS Inc, Chicago, Ill).

\section{Results}

\section{Factors Affecting the Pattern of Regional Lymph Node Involvement}

The following clinical and pathologic factors were analyzed to define any correlation with the level and type of N1 lymph node involvement: sex, type and duration of symptoms, anatomic site of the primary tumor, lobe of origin, clinical stage, histology, grading, and pT classification.

No statistical differences in the pattern of lymph node involvement were found for sex (male vs female, $P=.109$ ), type of symptoms (no symptoms vs cough vs chest pain vs hemoptysis vs other, $P=.063$ ), symptom duration (no symptoms vs $\leq 12$ weeks vs $>12$ weeks, $P=.133$ ), lobe of origin (right upper lobe vs middle lobe vs right lower lobe vs left upper lobe vs left lower lobe, $P=.085$ ), clinical 


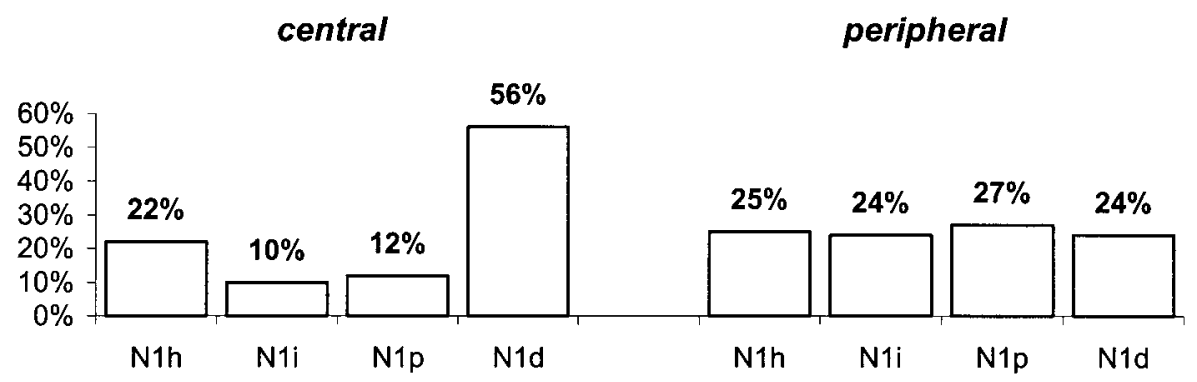

Figure 1. Correlation between the site of the primary tumor and the level of lymph node involvement $(P=.000)$.
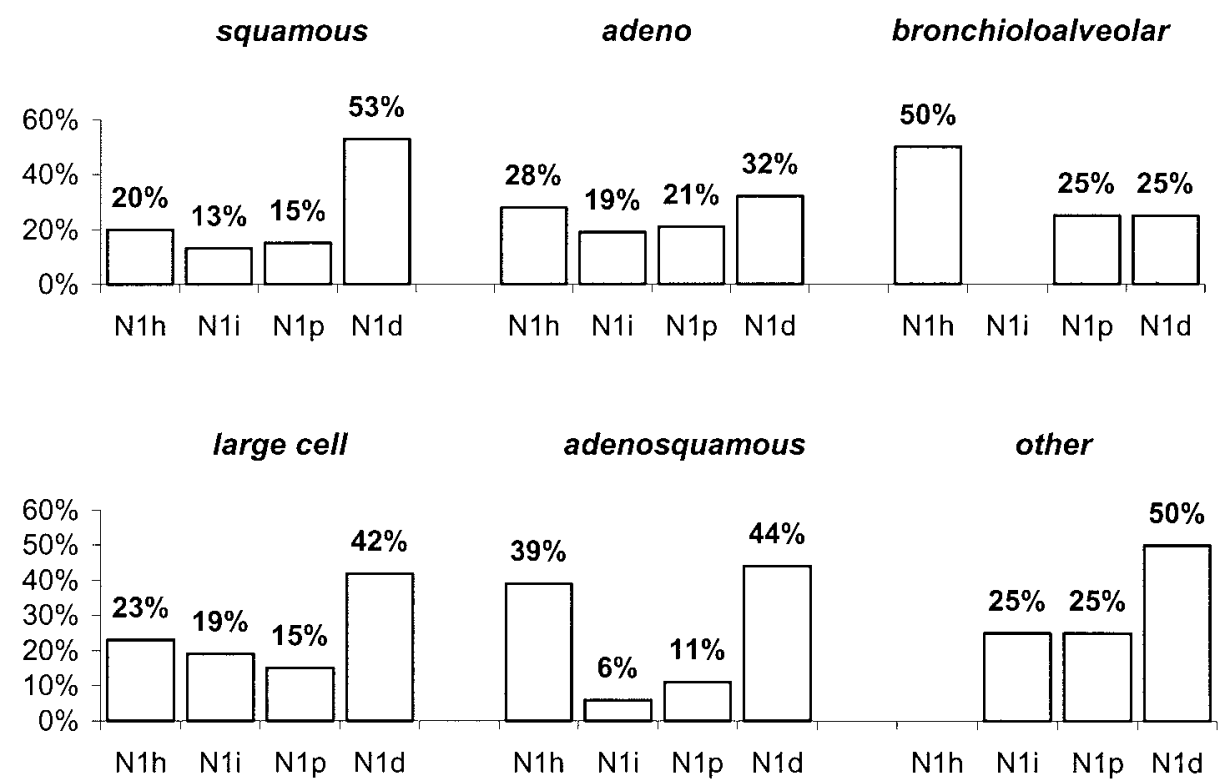

Figure 2. Correlation between the histology of the primary tumor and the level of lymph node involvement $(P=$ .027).

stage (stage I vs stage II vs stage III vs stage IV, $P=.108$ ), and grading (G1 vs G2 vs G3 vs G4, $P=.402$ ).

On the other hand, the Pearson $\chi^{2}$ test revealed a statistically significant correlation for the level and type of N1 involvement with the anatomic tumor site (central vs peripheral, $P=.000$ ), histology (squamous vs adenocarcinoma vs bronchioloalveolar vs large cell vs adenosquamous vs other, $P=.027$ ), pT classification (pT1 vs pT2 vs pT3 vs pT4, $P=.014$ ), and anatomic site (right vs left, $P=.039$ ). In other words, centrally located tumors tend to directly infiltrate neighboring lymph nodes, metastasize primarily to the hilar station, or both; peripherally located tumors showed no preference in the mechanism of lymphatic spread (Figure 1). Left-sided tumors prefer a direct invasion or tend to reach the hilar nodes more easily; lymph node involvement by right-sided tumors is well distributed among the different levels. SCC arises more frequently in the wall of the lobar bronchi and is associated with a higher incidence of direct lymph node involvement compared with the usually peripherally located adenocarcinoma (Figure 2). As we observed, the large proportion of patients with direct lymph node invasion can be explained by a majority of SCCs $(65 \%)$ in our population. Finally, the higher the pT class, the greater the probability of direct infiltration of lymph nodes, metastases to the hilar nodes, or both (Figure $3)$.

\section{Overall Survival of Patients With N1 Disease}

For all 535 patients with pN1 disease, the cumulative 3-, 5-, and 10 -year survivals were $51 \%, 40 \%$, and $27 \%$, respectively, with a median survival time of 38 months (Figure 4).

\section{Prognostic Factors for Patients With N1 Disease}

Survival curves were compared according to type and duration of symptoms, site of primary tumor, lobe of origin, 


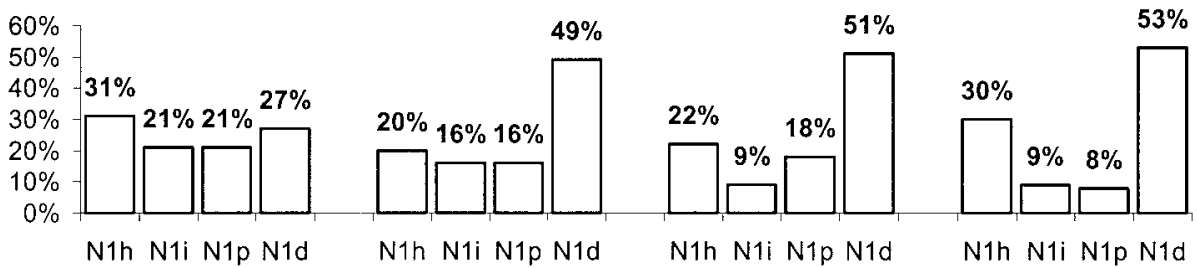

Figure 3. Correlation between pT classification and the level of lymph node involvement $(P=.014)$.

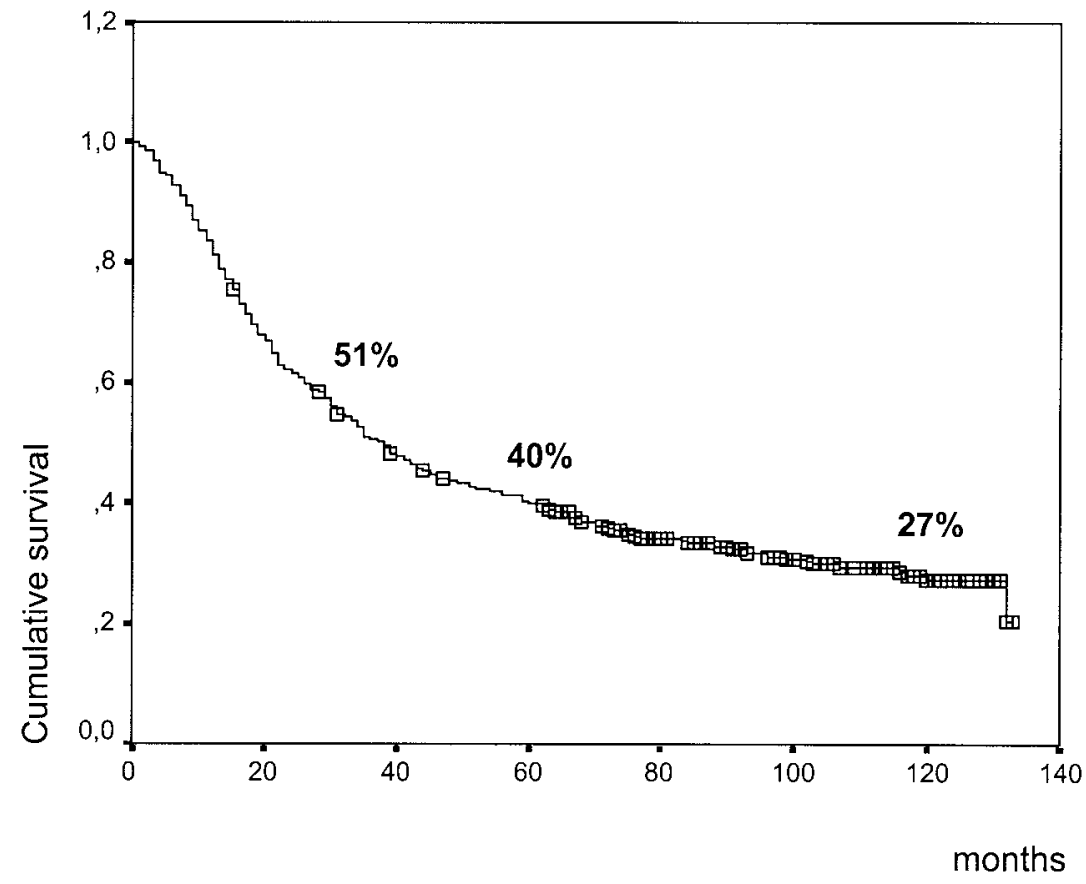

Figure 4. Actuarial overall survival for 535 patients with pN1 NSCLC (1990-1995).

pulmonary function (forced expiratory volume in 1 second $\left[\mathrm{FEV}_{1}\right]$ and percent of predicted $\mathrm{FEV}_{1}$ ), Karnofsky index, type of operation, blood transfusions, histology, pT classification, pattern of involvement of N1 lymph nodes (including number of involved lymph nodes and node stations), $\mathrm{R}$ status, and adjuvant treatment to individuate factors influencing the prognosis of patients with N1 disease (Table 3).

The log-rank test did not prove any significant correlation with the prognosis for type and duration of symptoms $(P=.4371$ and $P=.3717$, respectively), site of tumor $(P=$ $.8545)$, lobe of origin $(P=.2396), \mathrm{FEV}_{1}$ and percent of predicted $\mathrm{FEV}_{1}(P=.8354$ and $P=.2437$, respectively $)$, surgical procedure $(P=.2952)$, administration of blood transfusions $(P=.0666)$, and histology of the primary tumor $(P=.4833)$.

Seven factors showed, in the univariate analysis, a statistically significant correlation with the long-term outcome of the N1 disease: Karnofsky index, R status, pT classifi- cation, $\mathrm{N}$ status, number of involved lymph node levels, number of involved lymph nodes, and adjuvant treatment.

Patients were grouped as having a preoperative Karnofsky index of $100 \%, 90 \%$ to $80 \%$, or $70 \%$ or less. The actuarial 3-, 5- and 10-year survivals were 54\%, 45\%, and $35 \%$ for the former group; $50 \%, 41 \%$, and $28 \%$ for the intermediate group; and 44\%, 27\%, and $15 \%$ for the latter group, respectively $(P=.0181)$.

According to the $\mathrm{R}$ status, R0-resected patients had 3-, 5 -, and 10-year survivals of $56 \%, 45 \%$, and $31 \%$, respectively; R1-resected patients had 3-, 5-, and 10-year survivals of $31 \%, 20 \%$, and 14\%, respectively; and R2-resected patients had 3-, 5-, and 10-year survivals of 14\%, 14\% and $10 \%$, respectively $(P=.0000)$.

Pathologic T1 tumors showed a significantly better prognosis (3-, 5-, 10-year survivals of 79\%, 65\%, and 49\%, respectively) compared with pT2 tumors $(56 \%, 45 \%$, and $29 \%$, respectively), pT3 tumors $(38 \%, 29 \%$, and $20 \%$, re- 


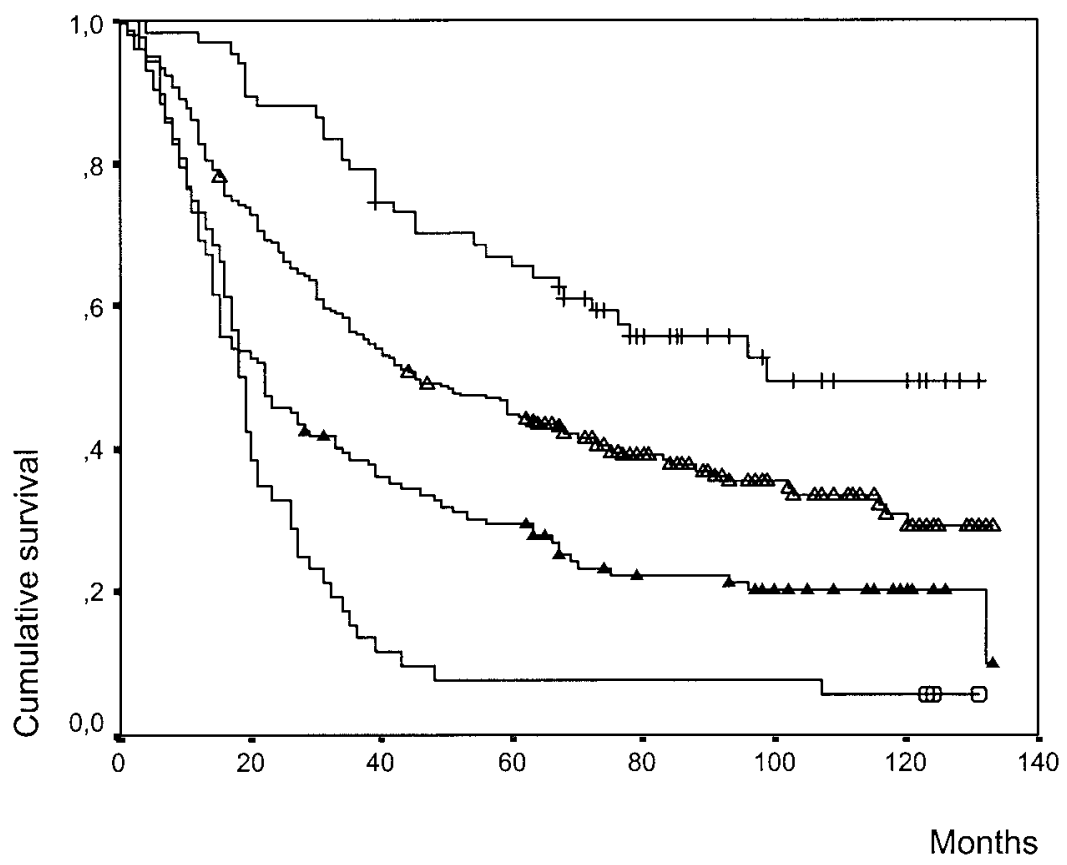

Figure 5. Survival curves of patients with N1 disease according to T classification: T1 (+), n = 67; T2 (open triangles), $\mathrm{n}=287$; $\mathrm{T3}$ (filled triangles), $\mathrm{n}=128$; T4 (open circles), $\mathrm{n}=53$ (log-rank test, $P=.0000)$.

spectively), and pT4 tumors $(13 \%, 8 \%$, and $6 \%$, respectively), with a log-rank test $P$ value of .0000 (Figure 5).

Survival curves according to the pattern of lymph node involvement also differed significantly $(P=.0184)$ : $55 \%$, $45 \%$, and $32 \%$ of patients with N1d disease were alive at 3 , 5 , and 10 years, respectively, whereas corresponding rates for patients with $\mathrm{N} 1 \mathrm{p}$ disease were $55 \%, 41 \%$, and $28 \%$; $46 \%, 39 \%$, and $27 \%$ for patients with N1i disease; and $42 \%$, $30 \%$, and $20 \%$ for patients with N1h disease, respectively (Figure 6). The results concerning the number of involved lymph nodes and nodal stations are described below. Among adjuvant untreated patients, survival at 3, 5, and 10 years was $58 \%, 47 \%$, and $32 \%$, respectively, and in the adjuvant radiotherapy group survival was $36 \%, 23 \%$, and $18 \%$, respectively; none of the 2 patients who received postoperative radiochemotherapy was alive at 3 years $(P=$ $.0000)$.

Surprisingly, in the multivariate analysis, neither the Karnofsky index, the number of involved lymph nodes and nodal levels, nor adjuvant therapy conserved statistical power $(P=.392, P=.566, P=.533$, and $P=.138$, respectively). The effect of $\mathrm{R}$ status on survival approached statistical relevance $(P=.055)$. The only independent factors able to influence the long-term prognosis of this population of patients were $\mathrm{pT}$ classification $(P=.000)$ and the type of lymph node involvement $(P=$ $.001)$.

\section{N1 Involvement and Prognosis}

The differentiated survival analysis according to pT classification demonstrated the prognostic power of the type of lymph node involvement only for T2 tumors, with 5-year survivals for the N1d, N1p, N1i, and N1h groups of 54\%, $40 \%, 42 \%$, and $29 \%$ and 10 -year survivals of $34 \%, 30 \%$, $31 \%$, and $16 \%$, respectively $(P=.0027)$. Thereafter, the pattern of N1 involvement showed a trend to influence prognosis in the population with $\mathrm{T} 3$ disease $(P=.0589)$. The outcome seemed to be independent from the type of N1 involvement in the early (T1, $P=.4536)$ and advanced stage (T4, $P=.2580)$.

In the overall population a multilevel lymph node metastasis, infiltration, or both correlated with a poorer prognosis compared with the involvement of a single station (5-year survivals: $27 \%$ vs $44 \%, P=.0002$ ).

We analyzed the importance of the number of involved lymph nodes independent of the mechanism of tumor invasion: the actuarial 5-year survivals for 1,2 to 5 , or more than 5 involved nodes were $49 \%, 40 \%$, and $30 \%$, respectively $(P=.0145)$.

\section{Cancer Recurrence}

We dispose of detailed information about the cancer-related clinical course of 358 (67\%) of 535 patients. Among them, $163(45 \%)$ patients had a cancer recurrence. The initial relapse site was distant in $106(65 \%)$ patients and locoregional in the remaining 57 (35\%) patients. The distribution 
TABLE 3. Univariate analysis of clinical-pathologic prognostic factors

\begin{tabular}{|c|c|c|c|c|c|}
\hline \multirow{2}{*}{\multicolumn{3}{|c|}{ nostic factors }} & \multirow{2}{*}{\multicolumn{2}{|c|}{ 5-y survival (\%) }} & \multirow[b]{2}{*}{$P$ value } \\
\hline & & & & & \\
\hline & 5-y survival (\%) & $P$ value & \multicolumn{3}{|l|}{ Histology } \\
\hline pT classification & & & Squamous & 42 & .4833 \\
\hline $\mathrm{T} 2$ & 45 & & Large cell & 38 & \\
\hline T3 & 29 & & Adenosquamous & 33 & \\
\hline T4 & 8 & & Other type & 50 & \\
\hline Radiotherapy & 23 & & $1.5-2.0$ & 39 & \\
\hline Chemoradiotherapy & 0 & & $<1.5$ & 36 & \\
\hline $\mathrm{R}$ status & & & Location & & \\
\hline RO & 44 & .0001 & Peripheral & 45 & .8545 \\
\hline R1 & 30 & & Central & 38 & \\
\hline
\end{tabular}

Involved node stations

Single station

Multilevel

No. of involved nodes

1

2-5

$>5$

$\mathrm{pN}$ classification

N1d

N1p

$\mathrm{N} 1 \mathrm{i}$

$\mathrm{N} 1 \mathrm{~h}$

Karnofsky Index

$100 \%$

$90-80 \%$

$\leq 70 \%$

Blood transfusions

No transfusion

Autologous blood

Eterologous blood

Site

Right

Left

Lobe

Upper

Middle

Lower

$\mathrm{FEV}_{1} \%$ predicted

$>70$

50-70

$<50$

Surgical procedure

Pneumonectomy

Lobectomy-bilobectomy

Segmentectomy

Duration of symptoms

No symptoms

$\leq 12 \mathrm{wk}$

$>12$ wk

Type of symptoms

No symptoms

Cough

Chest pain

Hemoptysis

Other
TABLE 3. Cont'd

.0002

.0145

.0184

.0181

.0666

.2113

.2396

.2437

.2952

.3717

.4371

of the site of first recurrence among the N1 groups (N1h vs N1i vs N1p vs N1d) differed significantly, with a prevalence of distant metastases and locoregional relapse for the N1h group of $41 \%$ and $12 \%$, respectively, and for the N1d group of $24 \%$ and $17 \%$, respectively $(P=.030)$. Among the 73 patients with locoregional recurrence, this arose despite previous systematic lymphadenectomy in the mediastinal lymph nodes in $35(48 \%)$ patients and from the bronchial stump in $38(52 \%)$ patients. No difference was observed in the distribution of the site of locoregional recurrence according to the type of nodal involvement $(P=.716)$. Among the 119 patients with distant metastases, the relapse target was the brain in $25(21 \%)$ patients, the lung in 25 (21\%) patients, bone in $22(18 \%)$ patients, the liver in 7 $(6 \%)$ patients, the adrenals in $1(1 \%)$ patient, other sites in $18(15 \%)$ patients, and synchronous multiple organs in 21 (18\%) patients. A significant difference was found in the distribution of the site of distant failure according to the type of nodal involvement $(P=.033$, Table 4$)$.

We calculated the probabilities of recurrence in patients with N1 tumors to be $21 \%$ within 1 year of treatment, $42 \%$ within 3 years, and $46 \%$ within 5 years.

\section{Discussion}

Although the NSCLC with metastases in the ipsilateral pulmonary lymph nodes $(\mathrm{N} 1$; ie, within the boundary of the pleural reflection) is commonly considered an early-stage disease, this group of patients experiences a disappointing prognosis after complete surgical resection, as stated by numerous authors. ${ }^{2,12-14}$ Mountain ${ }^{2}$ reported an overall survival at 5 years of approximately $40 \%$ for patients with $\mathrm{pN} 1$ disease compared with approximately $60 \%$ for patients with NO disease.

According to the UICC stage grouping, patients with stage IIA pT1N1 disease have a chance of 55\% to survive at 5 years, patients with stage IIB pT2 N1 disease of 39\%, patients with stage IIIA pT3 N1 disease of $25 \%$, and patients with stage IIIB pT4 N1 disease of approximately $8 \% .^{2}$ 


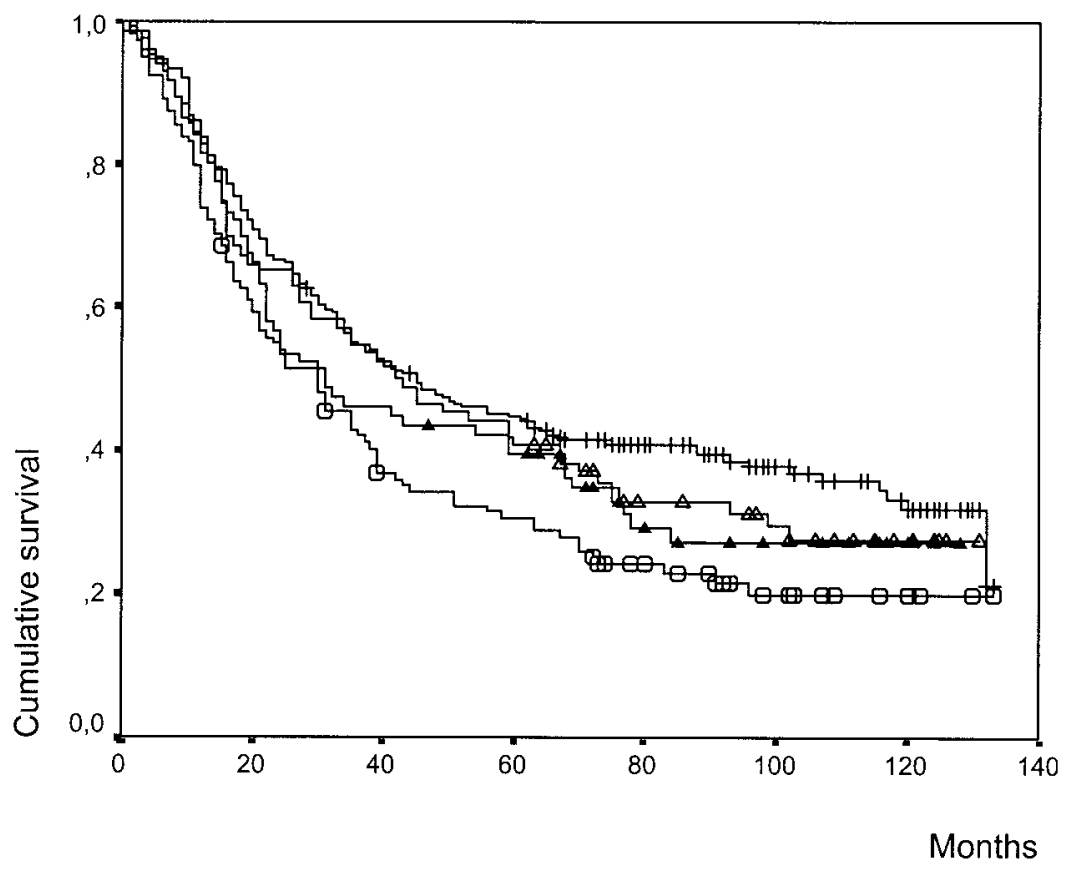

Figure 6. Survival curves of patients with N1 disease according to the level and type of lymph node involvement: N1d (+), $\mathrm{n}=$ 251; N1p (open triangles), $\mathrm{n}=87$; N1i (filled triangles), $\mathrm{n}=76$; Nih (open circles), $\mathbf{n}=342$ (log-rank test, $P=.0184$ ).

TABLE 4. Distribution of sites of distant failure according to type of lymph node involvement $(P=.033)$

\begin{tabular}{lcccc}
\hline & \multicolumn{4}{c}{ N1 type } \\
\cline { 2 - 5 } Site of distant recurrence & $\begin{array}{c}\text { Hilar } \\
(\mathbf{n}=\mathbf{7 3})\end{array}$ & $\begin{array}{c}\text { Interlobar } \\
(\mathbf{n}=\mathbf{5 0})\end{array}$ & $\begin{array}{c}\text { Lobar } \\
(\mathbf{n}=\mathbf{6 2})\end{array}$ & $\begin{array}{c}\text { Direct } \\
(\mathbf{n}=\mathbf{1 7 3})\end{array}$ \\
\hline Brain & 4 & 2 & 6 & 13 \\
Liver & 2 & - & 2 & 3 \\
Lung & 10 & 4 & 3 & 8 \\
Bone & 4 & 4 & 8 & 6 \\
Adrenals & 1 & - & - & - \\
Other & 2 & 2 & 5 & 9 \\
Multiple & 9 & 2 & 1 & 9 \\
\hline
\end{tabular}

Previous studies suggest that patients with ipsilateral pulmonary lymph node metastases represent a heterogeneous population, according to the level of lymph node involvement, as well as the mechanism of invasion (ie, lymph vessel spread or direct infiltration). ${ }^{3-7,14,15}$

Metastases to the hilar, interlobar, or both lymph nodes (stations 10 and 11, according to the new Regional Lymph Node Classification for Lung Cancer Staging) ${ }^{8}$ have been associated with a poorer prognosis compared with metastases in the intralobar lymph nodes (stations 12, 13, and 14). Moreover, the long-term outcome of the former group does not differ significantly from that of patients with singlestation mediastinal lymph node metastases (limited N2). $4,5,7,14,16$
We addressed our analysis to define the role of the level, as well as the mechanism, of lymph node involvement on the prognosis of patients with N1 disease. Our population represents a not negligible proportion of patients: $28 \%$ of all patients with operable NSCLC, with an incidence of 90 to 100 patients per year.

The importance of an accurate $\mathrm{N}$ staging has been stressed by several authors to avoid the Will Rogers phenomenon. $3,7,14,17,18$ The object of our analysis is a purified series because we accurately excluded N2 disease through routine cervical mediastinoscopy and an intraoperative systematic lymph node dissection of the upper and lower mediastinal compartment, as well as of the subcarinal station on both sides, and a dissection of subaortic and paraaortic nodes on left-sided thoracotomy.

The relatively large proportion of pneumonectomies (47\%) in our series compared with the smaller percentage (32\%) in the entire cohort of 1954 patients could be explained by considering that more than two thirds of tumors were located centrally; when feasible, a parenchyma-sparing procedure (ie, sleeve resection) has been preferred (9.3\% of all patients). The intraoperative decision to perform a pneumonectomy because of extranodal hilar, interlobar, or both metastases has been made in 29 (5.4\%) patients.

In our study we included patients with incomplete resection (R1 and R2); most of them (59/99 [60\%]) had microscopically diseased margins as a result of lymphangiosis in 
TABLE 5. Survival of patients with N1 NSCLC

\begin{tabular}{|c|c|c|c|c|c|c|c|c|}
\hline \multirow[b]{2}{*}{ Author } & \multirow[b]{2}{*}{ Year } & \multirow[b]{2}{*}{ No. } & \multirow[b]{2}{*}{ Stage } & \multicolumn{5}{|c|}{ 5-y survival (\%) } \\
\hline & & & & Global & N1h & N1i & N1p & N1d \\
\hline Ferguson and coworkers ${ }^{20}$ & 1986 & 34 & $\mathrm{~T} 1-2$ & 30.2 & - & - & - & - \\
\hline Maggi and coworkers ${ }^{19}$ & 1990 & 157 & T1-4 & 46.1 & - & - & - & - \\
\hline Martini and coworkers ${ }^{17}$ & 1992 & 214 & $\mathrm{~T} 1-2$ & 39 & - & - & - & - \\
\hline Yano and coworkers ${ }^{3}$ & 1994 & 78 & T1-3 & 49.2 & & & 64.5 & - \\
\hline Van Velzen and coworkers ${ }^{4}$ & 1996 & 57 & $\mathrm{~T} 1$ & 45.7 & & & 55.6 & 68.6 \\
\hline Van Velzen and coworkers 5 & 1997 & 369 & $\mathrm{~T} 2$ & 37.8 & & & 57.3 & 39.1 \\
\hline Van Velzen and coworkers 6 & 1999 & 111 & T3-4 & 27.2 & - & - & - & - \\
\hline Sawyer and coworkers ${ }^{21}$ & 1999 & 107 & T1-4 & 32 & - & - & - & - \\
\hline Riquet and coworkers ${ }^{14}$ & 1999 & 256 & $\mathrm{~T} 1-4$ & 47.5 & & & 53.6 & $\begin{array}{l}53.7(p) \\
27.3(h)\end{array}$ \\
\hline Yoshino and coworkers ${ }^{15}$ & 1999 & 43 & $\mathrm{~T} 1-2$ & 50.2 & & & 55 & - \\
\hline Asamura and coworkers ${ }^{7}$ & 2000 & 180 & $\mathrm{~T} 1-4$ & 67 & 54 & & & - \\
\hline Marra and coworkers & 2003 & 535 & T1-4 & 40 & 30 & 39 & 41 & 45 \\
\hline
\end{tabular}

$P$, Pulmonary, intralobar nodes; h, hilar, extralobar nodes.

${ }^{*} \mathrm{~N} 1 \mathrm{~h}$ and N1i were analyzed as a single group.

tN1i and $\mathrm{N} 1 \mathrm{p}$ were analyzed as a single group.

the hilar fat or peribronchial tissue. As expected, the multivariate survival analysis demonstrated a major role of residual tumor on long-term prognosis, although statistical relevance has not been completely reached $(P=.055)$.

The 5-year overall survival of patients with N1 disease varies in the literature between $27.2 \%$ and $67 \%$, according to the stage of disease (Table 5). ${ }^{3-7,14,15,17,19-21}$

The prognostic importance of lymph node involvement has been underlined by several authors. Yano and colleagues, ${ }^{3}$ in an analysis of 78 patients with pN1 NSCLC, found that the survival associated with lobar N1 disease was significantly better than that of patients with hilar-interlobar N1 disease $(64.5 \%$ vs $39.7 \%$ at 5 years, $P=.014)$. Van Velzen and associates ${ }^{4}$ studied the different subsets of the N1 population separately according to stage. In the first report on 58 patients with stage IIA pT1 N1 NSCLC, the 5-year survival of patients with $\mathrm{N} 1$ direct extension was superior to the survival of patients with $\mathrm{N} 1$ metastases (68.6\% vs $31.2 \%, P=.0038$ ), and the survival of patients with $\mathrm{N} 1$ disease by means of direct extension was better than the survival of patients with $\mathrm{N} 1$ hilar metastases $(P=$ .0006 ) but did not differ from the survival of patients with lobar metastases. Among 369 patients with stage IIB pT2 N1 NSCLC, the 5-year survival of patients with lobar metastases was superior to that of patients with hilar metastases (57.3\% vs $30.3 \%, P=.0028)$ and that of patients with lymph node involvement by means of direct invasion $(57.3 \%$ vs $39.1 \%, P=.03) .^{5}$ No significant effect on survival emerged in T3 N1 or T4 N1 tumors, the prognosis of which is related only to the completeness of the resection $(P=.0001){ }^{6}$

Riquet and coworkers ${ }^{14}$ reported 5 -year survival to be significantly better when $\mathrm{N} 1$ involvement was intralobar (levels 12 and 13, $\mathrm{n}=102$ ) compared with extralobar (hilar) involvement (levels 10 and $11, \mathrm{n}=154 ; 53.6 \%$ vs $38.5 \%$, $P=.02$ ). Survival was not related to the number of involved N1 stations or the type of involvement (direct extension or metastasis). A difference in the long-term outcome between hilar and lobar N1 disease failed to be demonstrated in the analysis of Maggi, ${ }^{19}$ Martini, ${ }^{17}$ Asamura, ${ }^{7}$ and their associates. Our data support the hypothesis that $\mathrm{N} 1$ disease is a compound of 2 subgroups: one metastasized outside the lobe and prognostically comparable with a limited N2 disease and the other confined inside the lobe by means of metastasis or direct invasion with a more favorable prognosis (5-year survivals of 30\%, 39\%, $41 \%$, and $45 \%$ for the N1h, N1i, N1p, and N1d groups, respectively; $P=.0184$ ).

The direct extension to adjacent lymph nodes has been associated with variable prognostic significance. Van Velzen and colleagues ${ }^{4}$ reported a highly significant difference between patients with direct invasion and those with hilar metastases for stage IIA pT1 N1 disease; on the other hand, they found comparable survivals between patients with direct extension and patients with hilar metastases in stage IIB pT2 N1 disease. ${ }^{5}$ These contrasting results might reflect the related level of lymph node invasion because direct extension concerns essentially intralobar N1 levels in T1 tumors and also hilar-interlobar N1 levels in T2 disease according to both the size and location of the primary tumor. The mechanism of lymph node invasion did not affect survival in the series of Riquet and colleagues. ${ }^{14}$ In the present series there was no difference in the survival curves of patients with direct lymph node involvement according to anatomic level $(P=.853)$, and the N1d disease related to a significantly better prognosis than the N1h disease $(P=.0184$, log-rank test; $P=.001$, Cox proportional hazard model).

Martini and colleagues ${ }^{17}$ observed the best survivals in 
patients with stage II NSCLC who had a single node involved $(P=.016)$ and tumors $3 \mathrm{~cm}$ or less in diameter $(P=$ .021 ), with a 5-year survival of $48 \%$ for patients with a combination of these favorable features. The administration of adjuvant radiotherapy lead to a worse prognosis $(P=$ .000035).

The role of the number of involved nodes on prognosis has been confirmed by our univariate analysis, with a more favorable long-term outcome when only one lymph node was colonized by tumor compared with multiple lymph node metastases $(P=.0145)$. Similarly, in our series singlestation $\mathrm{N} 1$ disease had a better prognosis than multilevel disease $(P=.0002)$.

Other prognostic factors that have been demonstrated to affect the outcome of patients with $\mathrm{N} 1$ disease were age, ${ }^{5} \mathrm{~T}$ stage, ${ }^{21}$ histology, ${ }^{6}$ visceral pleura involvement, ${ }^{5}$ positive bronchoscopic findings, ${ }^{21}$ the number of dissected lymph nodes, ${ }^{21}$ and macroscopic nodal metastases. ${ }^{15}$

The modality of recurrence seemed to be affected by the level and type of N1 involvement in the series of Yano and colleagues, ${ }^{3}$ with the brain being the most frequent site of distant metastasis in lobar N1 disease and the lungs being the most frequent site in hilar N1 disease. We also observed different patterns of cancer relapse according to nodal involvement: N1h tumors tended to recur at distant sites, with preference for the controlateral lung, whereas the most frequent target for N1d tumors was the brain $(P=.030)$.

In analyzing 58 patients with pT1 N1 NSCLC, Van Velzen and associates ${ }^{4}$ observed that relapses were more frequent in patients with hilar metastases than in patients with lobar metastases or with involvement by direct extension ( $56 \%$ vs $33 \%$ vs $22 \%$ ). In the hilar metastasis group the most common pattern of relapse was distant metastases. No difference in the modality of relapse on the basis of the level of N1 disease was detected by Riquet and colleagues. ${ }^{19}$ According to Martini and coworkers, ${ }^{17}$ local-regional recurrence was more frequent in patients with squamous carcinoma, whereas distant metastases were more commonly seen in adenocarcinomas (87\% of patients with metastases), with the brain as the most frequent site.

Surgical resection is considered thus far the treatment of choice for patients with N1 disease, but a great proportion of these patients experiences a cancer recurrence, either locoregional or distant. The best adjuvant treatment to associate with surgical resection is still a matter of debate.

The PORT meta-analysis reviewed data on 2128 patients with stage I to III disease and completely resected tumors from 9 randomized trials of postoperative radiotherapy versus surgery alone. The results showed a significant adverse effect of radiotherapy on survival, with a greater negative influence on patients with stage I to II, N0 to N1 disease. ${ }^{1}$ This could be explained by considering that the risk of systemic recurrence is dominant in all stages, minimizing the potential effect of radiotherapy on survival. The rationale for thoracic radiotherapy increases with advancing disease stage because the risk of intrathoracic recurrence increases. In patients with early-stage disease, the morbidity of radiotherapy outweighs the benefits regarding cancerspecific survival, leading to an overall detrimental effect. ${ }^{22}$

Our observation of a worse prognosis of patients treated with adjuvant radiotherapy compared with untreated patients (23\% vs $47 \% 5$-year survival, $P=.0001)$ confirms this opinion, although the unfavorable prognostic weight of radiotherapy disappeared on the multivariate analysis $(P=$ .138).

Adjuvant chemotherapy has been expected to gain survival improvement for patients with $\mathrm{N} 1$ disease. However, despite the compelling rationale for adjuvant systemic therapy in patients with resected node-positive NSCLC, it has been difficult to identify an effective regimen with present chemotherapeutic drugs. Randomized trials and meta-analyses of these trials have not found combined chemoradiotherapy to prolong survival in patients with completely resected stage I and II NSCLC..$^{23-26}$

Chemotherapy and chemoradiotherapy in a neoadjuvant setting are promising modalities, as shown by 2 randomized and several phase II clinical trials in locally advanced disease, ${ }^{27-30}$ but phase III studies in early-stage NSCLC are needed to define the optimal schedule and timing of systemic therapy. ${ }^{31}$

The present retrospective study confirms that N1 NSCLC is a compound of an intralobar subset that has a relatively more favorable prognosis and a hilar-interlobar subset that behaves like limited N2 disease. The direct infiltration of neighboring lymph nodes by the primary tumor tends to worsen the prognosis, as compared with NO NSCLC, and could be interpreted like a gate for the tumor to accede to the lymphatic circulation. Surgical resection remains the mainstay of therapy. Because the most common pattern of relapse after the operation is distant metastases in all groups, systemic therapy is mandatory to treat occult metastases and to provide survival advantages. The optimal timing, as well as the schedule of chemotherapy, should be investigated in future trials. As emerges from the PORT meta-analysis, ${ }^{1}$ radiotherapy could be able to prevent the occurrence of most local relapses, but toxicity could outweigh the benefits regarding cancer-specific survival in patients at relatively low risk for locoregional recurrence.

We acknowledge the assistance of Mrs Barbara Daut in data collection.

\section{References}

1. PORT Meta-analysis Trialists Group. Postoperative radiotherapy in non-small cell lung cancer: systematic review and meta-analysis of individual patient data from nine randomized controlled trials. Lancet. 1998;352:257-63. 
2. Mountain CF. Revisions in the international system for staging lung cancer. Chest. 1997;111:1710-7.

3. Yano T, Yokoyama H, Inoue T, Asoh H, Tayama K, Ichinose Y. Surgical results and prognostic factors of pathologic N1 disease in non-small-cell carcinoma of the lung. Significance of N1 level: lobar or hilar nodes. J Thorac Cardiovasc Surg. 1994;107:1398-402.

4. van Velzen E, Snijder RJ, Brutel de la Riviere A, Elbers HJ, van den Bosch JM. Type of lymph node involvement influences survival rates in T1N1M0 non-small cell lung carcinoma. Lymph node involvement by direct extension compared with lobar and hilar node metastases. Chest. 1996;110:1469-73.

5. van Velzen E, Snijder RJ, Brutel de la Riviere A, Elbers HR, van den Bosch JM. Lymph node type as a prognostic factor for survival in T2 N1 M0 non-small cell lung carcinoma. Ann Thorac Surg. 1997;63: 1436-40.

6. van Velzen E, de la Riviere AB, Elbers HJ, Lammers JW, van den Bosch JM. Type of lymph node involvement and survival in pathologic N1 stage III non-small cell lung carcinoma. Ann Thorac Surg. 1999;67:903-7.

7. Asamura H, Suzuki K, Kondo H, Tsuchiya R. Where is the boundary between N1 and N2 stations in lung cancer? Ann Thorac Surg. 2000;70:1839-46.

8. Mountain CF, Dresler CM. Regional lymph node classification for lung cancer staging. Chest. 1997;111:1718-23.

9. Fujimoto T, Zaboura G, Fechner S, Hillejan L, Schröder T, Marra A, et al. Completion pneumonectomy: current indications, complications, and results. $J$ Thorac Cardiovasc Surg. 2001;121:484-90.

10. World Health Organization. International histological classification of tumours. 2nd ed. Berlin: Springer-Verlag; 1988.

11. Kaplan EL, Meier P. Nonparametric estimation from incomplete observations. J Am Stat Assoc. 1958;53:457-81.

12. Mantel N. Evaluation of survival data and two new rank order statistics arising in its consideration. Cancer Chemother Rep. 1966;50:16370.

13. Cox DR. Regression models and life tables. J R Stat Soc B. 1972;34: $187-220$.

14. Riquet M, Manac'h D, Le Pimpec-Barthes F, Dujon A, Chehab A. Prognostic significance of surgical-pathologic N1 disease in non-small cell carcinoma of the lung. Ann Thorac Surg. 1999;67:1572-6.

15. Yoshino I, Nakanishi R, Osaki T, Takenoyama M, Taga S, Hanagiri T, et al. Unfavorable prognosis of patients with stage II non-small cell lung cancer associated with macroscopic nodal metastases. Chest. 1999;116:144-9.

16. Naruke T, Goya T, Tsuchiya R, Suemasu K. Prognosis and survival in resected lung carcinoma based on the new international staging system. J Thorac Cardiovasc Surg. 1988;96:440-7.

17. Martini N, Burt ME, Bains MS, McCormack PM, Rusch VW, Ginsberg RJ. Survival after resection of stage II non-small cell lung cancer. Ann Thorac Surg. 1992;54:460-6.

18. Keller SM, Adak S, Wagner H, Johnson DH. Mediastinal lymph node dissection improves survival in patients with stages II and IIIa non- small cell lung cancer. Eastern Cooperative Oncology Group. Ann Thorac Surg. 2000;70:358-66.

19. Maggi G, Casadio C, Mancuso M, Oliaro A, Cianci R, Ruffini E. Resection and radical lymphadenectomy for lung cancer: prognostic significance of lymphatic metastases. Int Surg. 1990;75:17-21.

20. Ferguson MK, Little AG, Golomb HM, Hoffman PC, DeMeester TR, Beveridge $\mathrm{R}$, et al. The role of adjuvant therapy after resection of $\mathrm{T} 1$ N1 M0 and T2 N1 M0 non-small cell lung cancer. J Thorac Cardiovasc Surg. 1986;91:344-9.

21. Sawyer TE, Bonner JA, Gould PM, Foote RL, Deschamps C, Lange $\mathrm{CM}$, et al. Factors predicting patterns of recurrence after resection of N1 non-small cell lung carcinoma. Ann Thorac Surg. 1999;68:1171-6.

22. Marks LB, Prosnitz LR. Postoperative radiotherapy for lung cancer: the breast cancer story all over again? Int J Radiat Oncol Biol Phys. 2000;48:625-7.

23. Wagner H. Postoperative adjuvant therapy for patients with resected non-small cell lung cancer: still controversial after all these years. Chest. 2000;117:110S-8S.

24. Bunn PA, Mault J, Kelly K. Adjuvant and neoadjuvant chemotherapy for non-small cell lung cancer. A time for reassessment? Chest. 2000;117:119S-22S.

25. Strauss GM. Role of chemotherapy in stages I to III non-small cell lung cancer. Chest. 1999;116:509S-16S.

26. Non-small Cell Lung Cancer Collaborative Group. Chemotherapy in non-small cell lung cancer: meta-analysis using updated data on individual patients from 52 randomized clinical trials. BMJ. 1995;311: 899-909.

27. Rosell R, Gomez-Codina J, Camps C, Maestre J, Padille J, Canto A, et al. A randomized trial comparing preoperative chemotherapy plus surgery with surgery alone in patients with non-small-cell lung cancer. N Engl J Med. 1994;330:153-8.

28. Roth JA, Fossella F, Komaki R, Ryan MB, Putnam JB Jr, Lee JS, et al. A randomized trial comparing perioperative chemotherapy and surgery with surgery alone in resectable stage IIIA non-small-cell lung cancer. J Natl Cancer Inst. 1994;86:673-80.

29. Eberhardt W, Wilke H, Stamatis G, Stuschke M, Harstrik A, Menker H, et al. Preoperative chemotherapy followed by concurrent chemoradiation therapy based on hyperfractionated accelerated radiotherapy and definitive surgery in locally advanced nonsmall cell lung cancer: mature results of a phase II trial. J Clin Oncol. 1998;16:622-34.

30. Stamatis G, Eberhardt W, Stuben G, Bildat S, Dahler O, Hillejan L. Preoperative chemoradiotherapy and surgery for selected non-small cell lung cancer IIIB subgroups: long-term results. Ann Thorac Surg. 1999;68:1144-9.

31. Pisters KM, Ginsberg RJ, Giroux DJ, Putnam JB, Kris MG, Johnson $\mathrm{DH}$, et al. Induction chemotherapy before surgery for early-stage lung cancer: a novel approach. Bimodality Lung Oncology Team. J Thorac Cardiovasc Surg. 2000;119:429-39. 